\title{
Distinct subcellular localization of E-cadherin between epithelioid angiomyolipoma and triphasic angiomyolipoma: A preliminary case-control study
}

\author{
XIN-GANG BI $^{1 *}$, LEI GUO ${ }^{2 *}$, XIAO-LIANG WANG ${ }^{2}$, QIAN WEI $^{2,3}$, QIANG DU $^{2}$, WEN-HAO JIANG ${ }^{4}$, \\ GUANG-YUAN ZHENG ${ }^{4}$, HONG-TU ZHANG ${ }^{2}$, JIAN-HUI MA ${ }^{1}$ and SHAN ZHENG ${ }^{2}$
}

Departments of ${ }^{1}$ Urology and ${ }^{2}$ Pathology, National Cancer Center/Cancer Hospital, Chinese Academy of Medical Sciences and Peking Union Medical College, Beijing 100021; ${ }^{3}$ Department of Pathology, Tibet Autonomous Region People's Hospital, Lhasa, Tibet Autonomous Region 850000; ${ }^{4}$ Department of Oncology, Beijing Second Hospital, Beijing 100031, P.R. China

Received May 18, 2016; Accepted March 17, 2017

DOI: $10.3892 / 01.2017 .6272$

\begin{abstract}
Epithelioid angiomyolipoma (EAML) is a rare variant of angiomyolipoma (AML). Previous studies have demonstrated that epithelial (E-)cadherin is expressed in two subtypes of AML, EAML and triphasic AML; however, the expression pattern of E-cadherin remains unclear. In the present study, a preliminary case-control study was conducted to determine the expression pattern of E-cadherin between EAML and triphasic AML, the control, focusing on the subcellular localization and expression category of E-cadherin. No significant difference was identified in the age, sex, history of tuberous sclerosis, smoking and alcohol consumption between the two groups $(\mathrm{P}>0.05)$. In EAML, 9 patients were categorized as exhibiting a low risk of malignant behavior and the other two were categorized as exhibiting an intermediate or high risk of malignant behavior. The proportion of cases expressing E-cadherin, human melanoma black-45 (HMB45), melanoma antigen recognized by $\mathrm{T}$ cells 1 (Mart1/Melan A), smooth muscle actin and progesterone receptor were 95.5 (21/22),
\end{abstract}

Correspondence to: Dr Shan Zheng, Department of Pathology, National Cancer Center/Cancer Hospital, Chinese Academy of Medical Sciences and Peking Union Medical College, 17 South Panjiayuan Lane, Beijing 100021, P.R. China

E-mail: zhengshan1002@aliyun.com

*Contributed equally

Abbreviations: AML, angiomyolipoma; CK, cytokeratin; EAML, epithelioid angiomyolipoma; EMA, epithelial membrane antigen; EMT, epithelial-to-mesenchymal transition; ECs, epithelioid cells; IHC, immunohistochemistry; IRS, immunoreactive score; MET, mesenchymal-to-epithelial transition; OR, odds ratio; PR, progesterone receptor; SD, standard deviation; SMA, smooth muscle actin; TSC, tuberous sclerosis complex

Key words: kidney, angiomyolipoma, epithelioid, epithelial cadherin, immunohistochemistry, case-control
$95.5(21 / 22), 86.4(19 / 22), 77.3(17 / 22)$ and $86.4 \%(19 / 22)$, respectively. E-cadherin was identified to be localized, using staining techniques, in the cell membrane and/or cytoplasm. The subcellular localization of E-cadherin was significantly different between EAML and triphasic AML; the majority of EAML cases revealed membranous and cytoplasmic staining, whereas triphasic AML cases demonstrated cytoplasmic staining $(\mathrm{P}=0.0093)$. The expression of $\mathrm{E}$-cadherin may be positively associated with HMB45 $(\mathrm{P}=0.0044)$ and Mart1/Melan A ( $\mathrm{P}=0.0049)$. The results of the present study identified that the subcellular localization of E-cadherin may be different between EAML and the control group of triphasic AML. Additionally, E-cadherin and melanocytic markers may be co-expressed in distinct subtypes of AML. A follow-up study with a large sample size to validate the results of the present study, followed by a mechanistic study based on cell lines to determine any significance, are warranted.

\section{Introduction}

Angiomyolipoma (AML) is the most common type of kidney mesenchymal tumor and accounts for between 1 and $3 \%$ of kidney tumors $(1,2)$. There are two clinical subtypes of AML: Sporadic AML and tuberous sclerosis complex (TSC)-associated AML (in patients with TSC) (3). The allelic loss of the TSC2 gene is a common genetic event in AML $(4,5)$. There are three histological subtypes of AML: Triphasic, monophasic and epithelioid (3). Triphasic AML, the most common subtype of AML, is a benign tumor containing various proportions of mature fat, thick-walled and poorly organized blood vessels and smooth muscle $(3,5)$. Monophasic and epithelioid AML (EAML) are classified as atypical AML (3). EAML is a rare subtype of AML which was first described by Mai et al (6) and is characterized by the proliferation of atypical epithelioid cells, with abundant eosinophilic and granular cytoplasm (5). Triphasic and EAML co-express melanocytic markers [human melanoma black-45 (HMB45), melanoma antigen recognized by $\mathrm{T}$ cells 1 (Mart1/Melan A), etc.] with variable intensity in smooth muscle markers, including smooth muscle actin 
(SMA), and progesterone receptor (PR) (5). In contrast with triphasic AML, $33 \%$ of EAML cases exhibit malignant potential with metastasis to the lymph nodes, liver, lungs or spine (5). However, there are no definite histopathological features to determine the prognosis of EAML. A number of genitourinary pathologists previously agreed that EAMLs should be divided into low, intermediate and high risk of malignant behavior (7-9). Currently, the majority of studies on malignant EAML have focused on the p53 gene and the protein Ki-67 (10-15).

One of the characteristic differences between EAML and triphasic AML is in the cellular morphology. The former is characterized by the appearance of epithelioid cells, which may exhibit the potency of metastasis, whereas the latter presents with spindle cells, which do not get the potency of metastasis (5). This difference may be similar to the process of epithelial-to-mesenchymal transition (EMT) and mesenchymal-to-epithelial transition (MET) to a certain extent $(16,17)$. Epithelial (E-)cadherin was reported as one of the key factors in EMT (16-18). A previous study conducted on renal epithelial tumor cell lines demonstrated that loss-of-function mutations of the TSC2 gene resulted in cytoplasmic localization of E-cadherin, which led to a decrease in cell-cell adhesion and the development of EMT (19). This may indicate that E-cadherin is an important factor in the progression of kidney tumors. In addition, the expression of E-cadherin is reported as a common event in AML, and, generally, the membrane immunoreactivity is more marked in epithelioid tumor cells compared with that in spindle tumor cells $(20,21)$. These results raise the question of whether EAML expresses E-cadherin with distinct subcellular localization, compared with triphasic AML.

In the present study, a preliminary case-control study was conducted to detect the expression of E-cadherin in EAML compared with triphasic AML (the most common AML subtype), as a control. The results of the present study demonstrated that the subcellular localization of E-cadherin in EAML was distinct from that in triphasic AML. It was additionally revealed that E-cadherin and melanocytic markers may be co-expressed in distinct subtypes of AML.

\section{Materials and methods}

Patients and tissues. The present study was part of a series of retrospective studies which was approved by the National Cancer Center Ethics Committee/Institutional Review Board (IRB; Chinese Academy of Medical Sciences and Peking Union Medical College, Beijing, China). All identifying information of patients was anonymized, and there were no risks anticipated to the participants. It was difficult to acquire patient consent from each participant in a retrospective study and, with the permission of the National Cancer Center Ethics Committee/IRB, patient consent was waived in this series of studies. There were 9 males and 13 females recruited into the present study. The median age was 42.8 , and ranged from 23-58 years old.

In the present study, 11 cases of EAML from the National Cancer Center/Cancer Hospital, Chinese Academy of Medical Sciences and Peking Union Medical College, between January 2005 and December 2011. Another 11 cases of triphasic
AML, during the same period, were selected as the control. The World Health Organization classification of renal tumors (2004) criteria were applied to all EAML and classic AML cases examined in the present study (5). EAML followed these diagnostic criteria, as follows: i) Polygonal larger cells with abundant granular cytoplasm; ii) enlarged vesicular nuclei, prominent nucleoli, multinucleated or enlarged ganglion-like cells; iii) nuclear atypia; iv) expression of one or both melanocytic markers (HMB-45, Mart1/Melan A); v) positive or negative for SMA or PR; and vi) negative immunoreaction for the epithelial markers pan-cytokeratin (CK), CK8, CK18 and epithelial membrane antigen (EMA). The cases of triphasic AML followed these diagnostic criteria, as follows: i) A variable mixture of fat, blood vessels and smooth muscle; ii) the smooth cells emanated from blood vessel walls in a radical fashion with or without atypia; iii) mature adipose tissue and thick-walled poorly organized blood vessels were observed in the tumor; and iv) immunoprofile was similar to that of EAML (as aforementioned).

In the EAML group, the epithelioid component was required to be $\geq 5 \%$ in the tumors, which used the same criteria as a previous study (7). The nuclear atypia was also required to be at least moderate in EAML. The nuclear atypia criteria followed the criteria of Brimo et al (7). The nuclear atypia was defined as vesicular nuclei, prominent nucleoli and nuclear size, which was at least twice the size of adjacent nuclei. Moderate atypia described epithelioid cells that were intermediate in size and exhibited enlarged nuclei with moderate pleomorphism and prominent nucleoli. Identifying carcinoma-like growth patterns was conducted following the definition of Nese et al (8): Tumor cells arranged as cohesive nests, broad alveoli and compartmentalized sheets. All EAMLs were divided into three groups of malignant behaviors (low, intermediate and high risk) on the basis of five adverse prognostic parameters: Associated TSC of concurrent AML, necrosis, tumor size $>7 \mathrm{~cm}$, extrarenal extension and/or renal vein involvement and a carcinoma-like growth pattern $(8,9)$. Tumors with $<2$ adverse prognostic parameters were classified as low risk, tumors with between 2 and 3 adverse prognostic parameters were classified as intermediate risk and tumors with $\geq 4$ adverse prognostic parameters were classified as high risk of exhibiting malignant behavior.

Three pathologists (Y.Z., H.T.Z. and S.Z.) from the Department of Pathology, National Cancer Center/Cancer Hospital, Chinese Academy of Medical Sciences and Peking Union Medical College (Beijing, China) reviewed all the hematoxylin and eosin slices of every case to evaluate the proportion of epithelioid cells (ECs), nuclear atypia and growth pattern. The demographic and clinical information including age, sex, history of TSC, smoking and alcohol consumption was obtained from medical records. Tumor size was obtained according to the gross description of the specimen following formalin fixation.

Immunohistochemistry (IHC). IHC staining was performed in all 22 cases using 9 antibodies (Table I) according to the manufacturer's protocol for each antibody. Tissue blocks with tumor and normal tissue were selected for the IHC staining. Prior to IHC, sections of $4 \mu \mathrm{m}$ from each block were deparaffinized by xylene, rehydrated by gradient alcohol and then steamed 
Table I. Antibody information.

\begin{tabular}{|c|c|c|c|c|c|}
\hline Antibody & Clone & $\begin{array}{l}\text { Catalogue } \\
\text { numbers }\end{array}$ & Dilution & $\begin{array}{l}\text { Incubation } \\
\text { time, min }\end{array}$ & Source \\
\hline CK8 & TS1 & Kit-0034 & $\begin{array}{l}\text { Working } \\
\text { solution }\end{array}$ & 30 & $\begin{array}{l}\text { Fuzhou Maixin Biotech Co., Ltd., } \\
\text { Fuzhou, China }\end{array}$ \\
\hline CK18 & DC10 & MAB-0182 & $\begin{array}{l}\text { Working } \\
\text { solution }\end{array}$ & 20 & Fuzhou Maixin Biotech Co., Ltd. \\
\hline E-cadherin & $4 \mathrm{~A} 2 \mathrm{C} 7$ & MAB-0589 & $\begin{array}{l}\text { Working } \\
\text { solution }\end{array}$ & 30 & Fuzhou Maixin Biotech Co., Ltd. \\
\hline EMA & E29 & IR62961 & $\begin{array}{l}\text { Working } \\
\text { solution }\end{array}$ & 30 & $\begin{array}{l}\text { Dako; Agilent Technologies, Inc., } \\
\text { Santa Clara, CA, USA }\end{array}$ \\
\hline Pan-keratin & AE1/AE3 & Z2061 & $1: 120$ & 30 & Zeta Corporation, Arcadia, CA, USA \\
\hline Mart1/Melan A & A103 & IR63361 & $\begin{array}{l}\text { Working } \\
\text { solution }\end{array}$ & 20 & Dako; Agilent Technologies, Inc. \\
\hline Melanoma & HMB45 & MAB-0098 & $\begin{array}{l}\text { Working } \\
\text { solution }\end{array}$ & 30 & Fuzhou Maixin Biotech Co., Ltd. \\
\hline PR & $1 \mathrm{E} 2$ & $790-4296$ & $\begin{array}{l}\text { Working } \\
\text { solution }\end{array}$ & 30 & $\begin{array}{l}\text { Ventana Medical Systems, Inc., } \\
\text { Tucson, AZ, USA }\end{array}$ \\
\hline SMA & $1 \mathrm{~A} 4$ & 202M-97 & $\begin{array}{l}\text { Working } \\
\text { solution }\end{array}$ & 30 & Cell Marque, Austin, TX, USA \\
\hline
\end{tabular}

CK, cytokeratin; E-cadherin, epithelial cadherin; EMA, epithelial membrane antigen; Mart1/Melan A, melanoma antigen recognized by T cells 1; PR, progesterone receptor; SMA, smooth muscle actin.

in $10 \mathrm{mM}$ sodium citrate buffer, $\mathrm{pH} 6.0$ (except SMA), for $2 \mathrm{~min}$ in a pressure cooker. SMA did not require any antigen retrieval according to the manufacturer's protocol. Endogenous peroxidase was blocked by incubating the sections at room temperature with ultraView Universal diaminobenzidine (DAB) inhibitor $\left(3 \% \mathrm{H}_{2} \mathrm{O}_{2}\right.$; Ventana Medical Systems, Inc., Tucson, AZ, USA) for $5 \mathrm{~min}$. Sections were subsequently incubated with primary antibodies for between 20 and 30 min according to the antibody (Table I). Following this, sections were incubated in the ultraView Universal horseradish peroxidase Multimer for $15 \mathrm{~min}$ and then visualized using ultraView Universal DAB Chromogen (0.2\% DAB), ultraView Universal DAB $\mathrm{H}_{2} \mathrm{O}_{2}$ and ultraView Universal DAB Copper (all Ventana Medical Systems, Inc.).

The results of staining were initially assigned an immunoreactive score (IRS) $(22,23)$. Values for the proportion of positive cells were determined as follows: Negative, $0 ; 1-10 \%$, $1 ; 11-50 \%, 2 ; 51-80 \%, 3$; and 81-100\%, 4. Staining intensity was qualitatively evaluated as follows: Negative, 0 ; weak, 1; moderate, 2; and strong, 3. The final IRS was calculated by multiplying the value for the proportion of positive cells by the value for staining intensity. The total range of values was 0-12. Subsequently, the expression of each biomarker was divided into four categories based on the IRS: IRS 0, negative (-); IRS 1-4, weak staining (+); IRS 5-8, intermediate staining (++); IRS 9-12, strong staining (+++). The slices were evaluated independently by three pathologists (X.L.W., Q.W. and S.Z.). In ambiguous cases, these three pathologists evaluated together using a Leica Multiviews system (Leica Microsystems, Inc., Buffalo Grove, IL, USA) to obtain the final result.
Statistical analysis. The mean and standard deviation (SD) were calculated for continuous data and a Student's t-test was carried out. Frequency and proportion values were reported for the categorical data, including sex, surgical type, history of TSC, smoking and alcohol history. Fisher's exact test was conducted. Spearman's rank correlation was used to determine the association between E-cadherin and other markers of interest, including HMB45, Mart1/Melan A, SMA and PR. Odds ratios (ORs) and 95\% confidence intervals for risk were calculated. To estimate the adjusted OR, the logistic regression models including age, sex and tumor size as factors were used. All the statistical tests carried out were two-tailed and $\mathrm{P}<0.05$ was considered to indicate a statistically significant difference. No adjustment for multiplicity was applied since all analyses were to be exploratory. In EAML, only the staining result of epithelial components was compared with that in the control (triphasic AML) components. The data were analyzed using SAS (version 9.2; SAS Institute Inc., Cary, NC, USA).

\section{Results}

Baseline characteristics between EAML and control group of triphasic $A M L$. There were a total of 22 cases in the EAML group and the control (triphasic AML) group, some of which were reported previously (24) (Table II). Only 1 case with EAML exhibited metastases (24). In total, there were 13 females and 9 males with a mean \pm SD age of $42.8 \pm 9.8$ years. Tumor size ranged between 2.0 and $17.5 \mathrm{~cm}$ and the majority of cases underwent radical nephrectomy. In the EAML group, 9 cases exhibited $\geq 90 \%$ ECs in tumors; the other two cases exhibited $10 \%$ ECs in tumors. None of the patients had a 


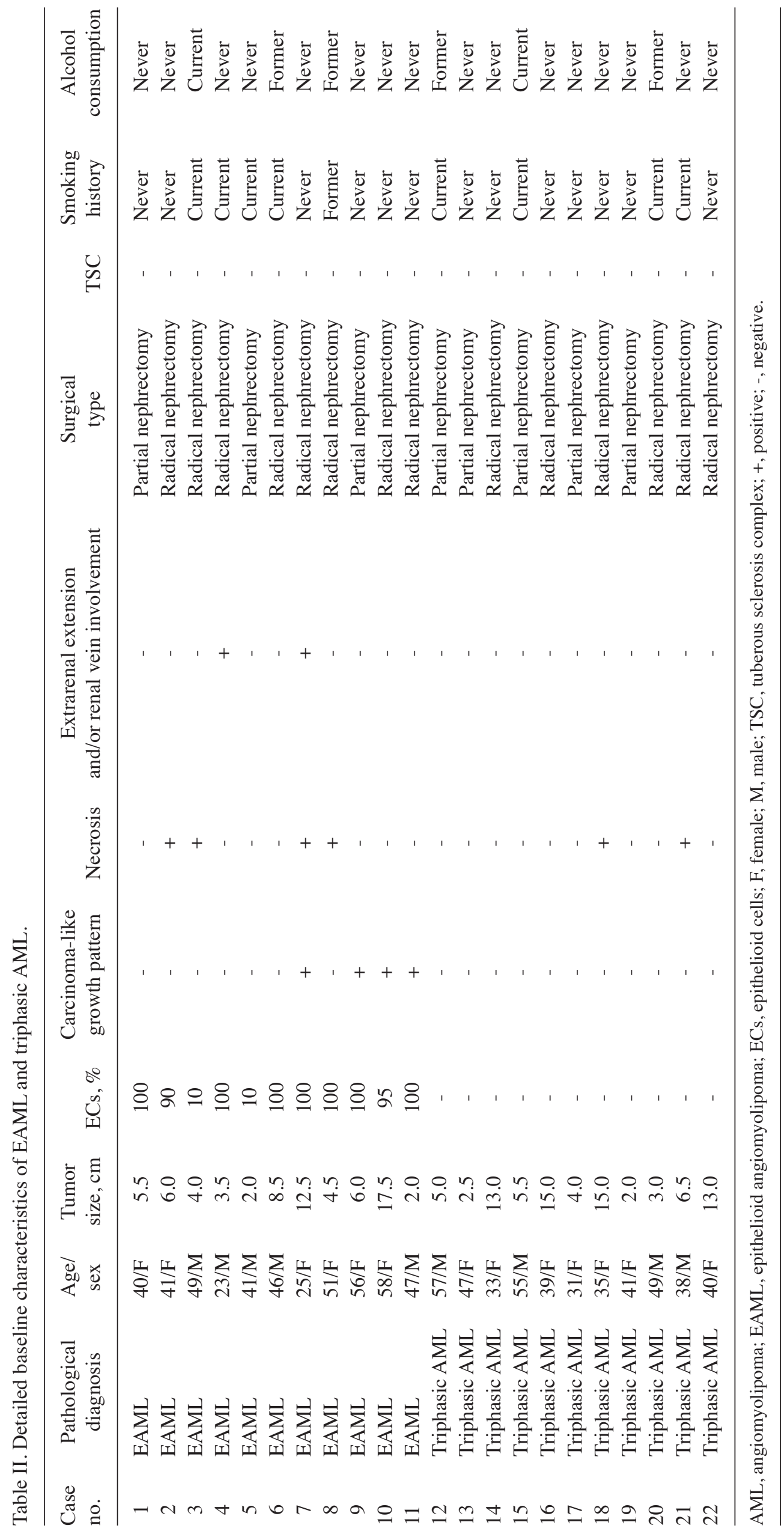


Table III. Comparison of baseline characteristics between EAML and triphasic AML.

\begin{tabular}{|c|c|c|c|}
\hline Characteristic & EAML & Triphasic AML & Total \\
\hline \multicolumn{4}{|l|}{ Age, years } \\
\hline $\mathrm{n}$ & 11 & 11 & 22 \\
\hline Mean (SD) & $43.4(11.2)$ & $42.3(8.7)$ & $42.8(9.8)$ \\
\hline Range & $23-58$ & $31-57$ & $23-58$ \\
\hline \multicolumn{4}{|l|}{ Sex } \\
\hline Female, n (\%) & $6(54.5)$ & $7(63.6)$ & $13(59.1)$ \\
\hline Male, n (\%) & $5(45.5)$ & $4(36.4)$ & $9(40.9)$ \\
\hline \multicolumn{4}{|l|}{ Tumor size, $\mathrm{cm}$} \\
\hline Mean (SD) & $6.5(4.7)$ & $7.7(5.2)$ & $7.0(4.9)$ \\
\hline Range & $2.0-17.5$ & $2.0-15.0$ & $2.0-17.5$ \\
\hline \multicolumn{4}{|l|}{ Surgical type } \\
\hline Radical nephrectomy, n (\%) & $8(72.7)$ & $6(54.5)$ & $14(63.6)$ \\
\hline Partial nephrectomy, n (\%) & $3(27.3)$ & $5(45.5)$ & $8(36.4)$ \\
\hline \multicolumn{4}{|l|}{ History of TSC } \\
\hline Yes, n (\%) & $0(0)$ & $0(0)$ & $0(0)$ \\
\hline No, n $(\%)$ & $11(100)$ & $11(100)$ & $22(100)$ \\
\hline \multicolumn{4}{|l|}{ Smoking history } \\
\hline Never, n (\%) & $6(54.5)$ & $7(63.6)$ & $13(59.1)$ \\
\hline Former or current, n (\%) & $5(45.5)$ & $4(36.4)$ & $9(40.9)$ \\
\hline \multicolumn{4}{|l|}{ Alcohol consumption } \\
\hline Never, n (\%) & $8(72.7)$ & $8(72.7)$ & $16(72.7)$ \\
\hline Former or current, n (\%) & $3(27.3)$ & $3(27.3)$ & $6(27.3)$ \\
\hline
\end{tabular}

AML, angiomyolipoma; EAML, epithelioid angiomyolipoma; SD, standard deviation; TSC, tumor sclerosis complex.

history of TSC; however, 15 of the patients had a history of smoking and/or alcohol consumption. The important baseline characteristics between EAML and control groups were comparable ( $\mathrm{P}>0.05$; Table III). In the EAML group, 9 cases were classified as possessing a low risk of malignant behavior, 1 case was classified as possessing an intermediate risk of malignant behavior and 1 case was classified as possessing a high risk of malignant behavior (Table IV).

Expression of E-cadherin and other biomarkers in all AML. First, the expression of 9 biomarkers in the two AML groups were identified (Figs. 1 and 2). In all 22 cases, the proportion of cases expressing E-cadherin, HMB45, Mart1/Melan A, SMA and PR was 95.5 (21/22), 95.5 (21/22), 86.4 (19/22), 77.3 (17/22) and $86.4 \%$ (19/22), respectively. All biomarkers, with the exception of PR, exhibited positive subcellular localization in the cell membrane and/or plasma. The latter exhibited nuclear staining. The majority of EAML cases $(8 / 11,72.7 \%)$ demonstrated intermediate E-cadherin expression, whereas that of the control cases $(8 / 11,72.7 \%)$ demonstrated weak E-cadherin expression. In all cases, results demonstrated that E-cadherin and HMB45 were expressed; however, E-cadherin and Mart1/Melan A were expressed in 86.4\% (19/22) of cases (Table IV). AE1/AE3, CK18, CK8 and EMA were not expressed in any of the cases (Table IV).

The association between E-cadherin and other biomarkers was subsequently identified. The results from the present study revealed that the expression of E-cadherin demonstrated a positive association with the expression of HMB45 $(\mathrm{P}=0.0044$; Table V) and Mart1/Melan A ( $\mathrm{P}=0.0049$; Table V). Additionally, the results of the present study revealed negative associations between E-cadherin and SMA or PR. However, none of these results were considered to indicate a statistical significance.

Distinct subcellular localization of E-cadherin in EAML and control group of triphasic AML. The subcellular localization and staining categories of E-cadherin were investigated between EAML and the control group of triphasic AML to observe any differences. The majority of cases in the EAML group demonstrated cell membrane and cell plasma staining of E-cadherin (8/11), whereas the majority of cases in the triphasic AML group demonstrated cell plasma staining only (9/11). The subcellular localization of E-cadherin was identified to demonstrate differences between the EAML group and the control group $(\mathrm{P}=0.0089)$. The $\mathrm{OR}$ was calculated to be 20.0 , indicating that cases with cell membrane E-cadherin staining (with or without cell plasma staining) had a 20 -fold increased likelihood of exhibiting EAML rather than triphasic AML (Table VI). However, no statistically significant difference in staining categories of E-cadherin between these two groups was identified $(\mathrm{P}=0.0950)$ following the adjustment for age, sex and tumor size (data not shown). 


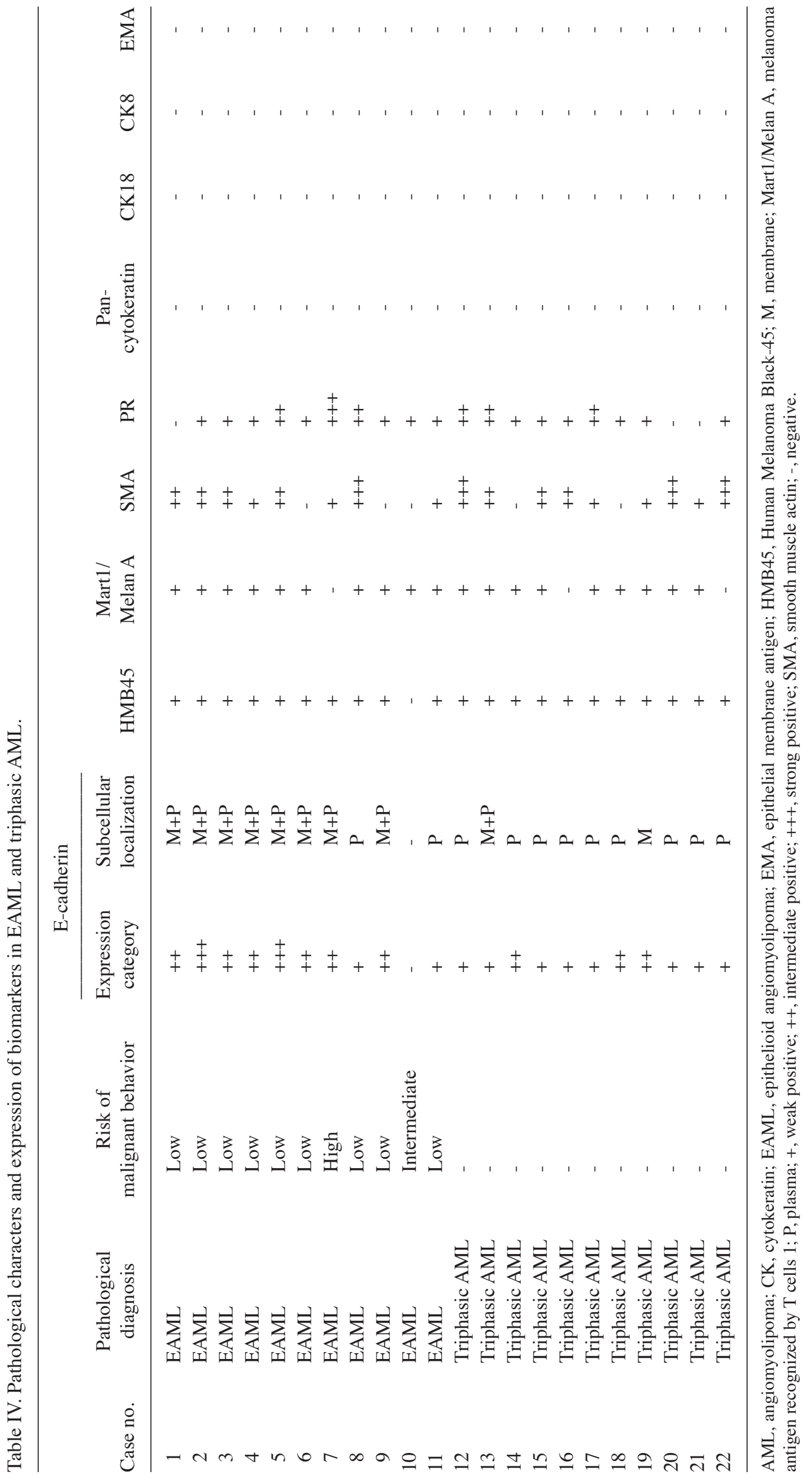



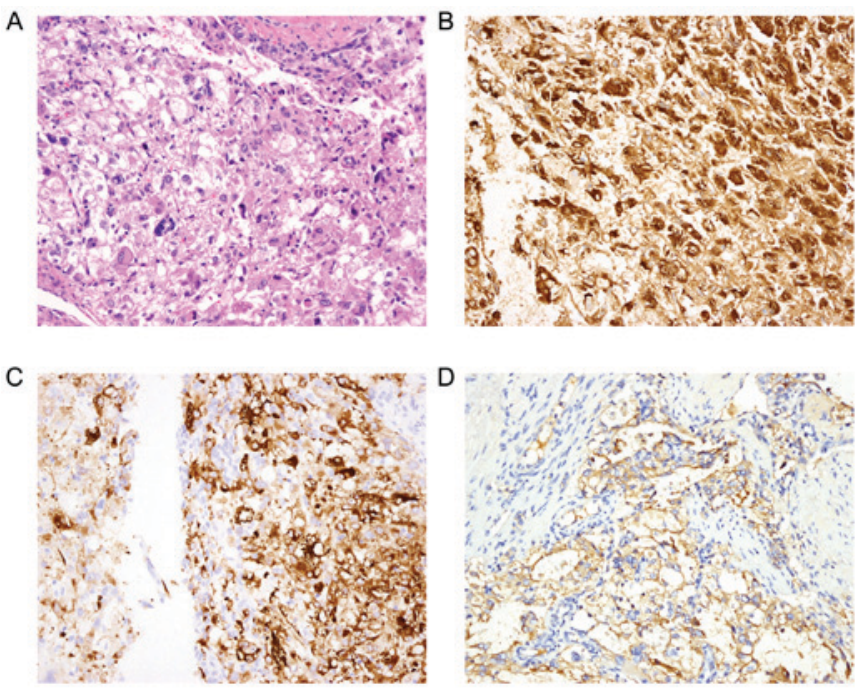

Figure 1. Hematoxylin and eoisin staining demonstrating the histopathological features and the expression of biomarkers (magnification, x200) in EAML. (A) Histopathological features of EAML. Polygonal larger cells with abundant granular cytoplasm and nuclear atypia, prominent nucleoli and multinucleated cells. (B) Human Melanoma Black-45 staining for EAML (strong plasma staining). (C) Melanoma antigen recognized by T cells 1 for EAML (strong plasma staining). (D) Epithelial cadherin staining for EAML (middle membrane combined with plasma staining). EAML, epithelioid angiomyolipoma.
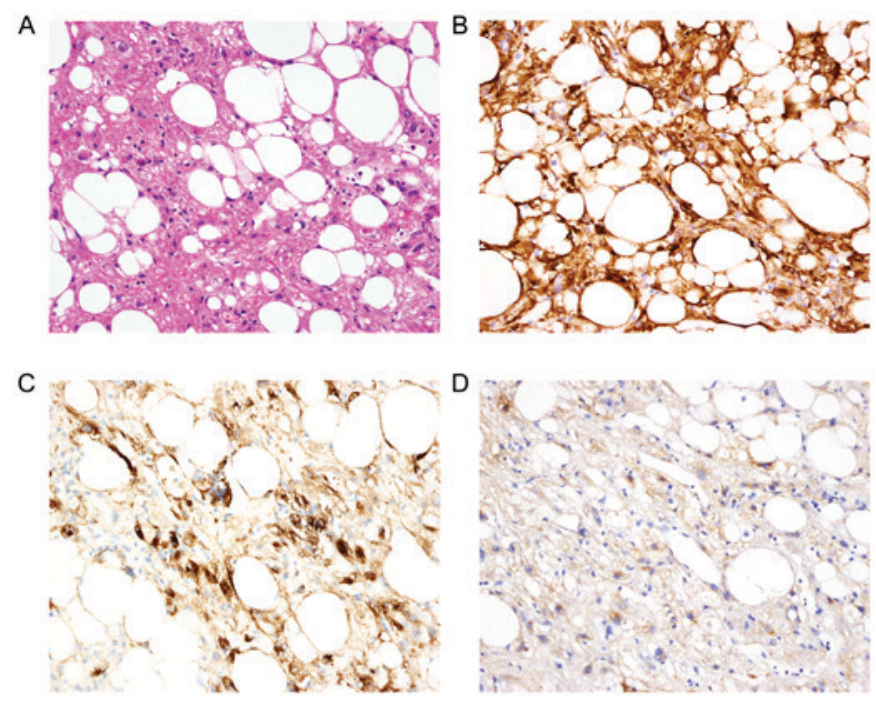

Figure 2. Hematoxylin and eoisin staining demonstrating the histopathological features and the expression of biomarkers in AML (magnification, x200). (A) Histopathological features of AML, fat and smooth muscle cells (B) Human Melanoma Black-45 staining for AML (strong plasma staining). (C) Melanoma antigen recognized by T cells 1 staining for AML (moderate plasma staining). (D) Epithelial cadherin staining for AML (weak plasma staining). AML, angiomyolipoma.

The majority of cases classified as possessing a low risk of malignant behavior exhibited at least intermediate E-cadherin staining $(7 / 9,77.8 \%)$, whereas the case classified as possessing an intermediate risk of exhibiting malignant behavior exhibited negative E-cadherin staining. The case classified as exhibiting a high risk of malignant behavior group also exhibited intermediate E-cadherin staining; however, due to the limited number of cases, the significance of this remains unclear.
Table V. Spearman's rank correlation between E-cadherin and other biomarkers.

\begin{tabular}{llccc}
\hline Variable & Biomarker & $\mathrm{n}$ & $\begin{array}{c}\text { Sample } \\
\text { correlation }\end{array}$ & P-value \\
\hline E-cadherin & HMB45 & 21 & 0.5956 & 0.0044 \\
& Mart1/Melan A & 22 & 0.5777 & 0.0049 \\
& SMA & 22 & -0.0904 & 0.6893 \\
& PR & 22 & -0.1879 & 0.4023 \\
\hline
\end{tabular}

E-cadherin, epithelial cadherin; HMB45, human melanoma black-45; Mart1/Melan A, melanoma antigen recognized by T cells 1; SMA, smooth muscle actin; PR, progesterone receptor.

\section{Discussion}

E-cadherin belongs to the classical cadherin superfamily; it has five repetitive extracellular cadherin domains and a conserved cytoplasmic domain $(25,26)$. E-cadherin was first reported as a cell adhesion protein in chicken liver cells and mice $(27,28)$, and Takeichi and colleagues named this $\mathrm{Ca}^{2+}$-dependent cell-cell adhesion molecule E-cadherin in the early 1980s (29,30). Since then, the role E-cadherin serves in cell architecture and tissue formation of normal epithelium has been extensively investigated $(25,26,31,32)$. It has been identified previously that E-cadherin is a key factor in EMT and MET (33). EAML exhibits epithelioid cells with metastatic potential, while triphasic AML presents with spindle cells without metastatic potential (5). These differences between EAML and triphasic AML were similar to the EMT process to a certain extent $(16,17)$. Hence, the factors associated with the EMT process, including E-cadherin (33), may be a reason for these differences. Previous studies have revealed E-cadherin to be expressed in tumors of the urinary system $(34,35)$, soft tissue sarcoma $(36,37)$ and AML $(20,21)$. E-cadherin may be associated with the differences in cellular morphology between EAML and triphasic AML. The present preliminary case-control study was conducted to detect the expression pattern of E-cadherin in EAML compared with the common subtype of AML, triphasic AML.

The results of the present study indicated that E-cadherin was expressed commonly in EAML and triphasic AML. All but one case expressed E-cadherin and half of the cases demonstrated at least intermediately stained E-cadherin. The proportion of cases demonstrating E-cadherin expression in EAML was slightly decreased compared with that in triphasic AML. The results of the present study were similar to those from a previous study by Wang et al (20) which revealed a slight increase in the proportion of cases demonstrating E-cadherin expression (98\%), and the majority of cases $(71 \%)$ demonstrated moderate or strong E-cadherin staining. The slight difference between the two studies may be due to the differences in case selection. In the present study, renal EAML and control (triphasic AML) cases were selected at the constituent ratio 1:1. Conversely, the study by Wang et al (20) collected a variety of AML types (including renal, hepatic and retroperitoneal), with only 2 cases of EAML (5\%). The 
Table VI. Fisher's exact test, OR for E-cadherin score and localization between EAML and triphasic AML.

\begin{tabular}{lcccc}
\hline $\begin{array}{l}\text { E-cadherin } \\
\text { expression }\end{array}$ & $\begin{array}{c}\text { EAML, } \\
\mathrm{n}(\%)\end{array}$ & $\begin{array}{c}\text { Triphasic } \\
\text { AML, }(\%)\end{array}$ & $\begin{array}{c}\text { P-value } \\
\text { (Fisher's exact test) }\end{array}$ & $\begin{array}{c}\text { OR (95\% CI), } \\
\text { P-value }\end{array}$ \\
\hline $\begin{array}{l}\text { E-cadherin localization } \\
\text { Membrane staining with or without plasma }\end{array}$ & $8(80.0)$ & $2(18.2)$ & 0.0089 & $20.0(2.0,159.1), 0.0093$ \\
$\begin{array}{l}\text { Cytoplasm only } \\
\begin{array}{l}\text { E-cadherin expression category } \\
++/+++\end{array}\end{array}$ & $\begin{array}{l}2(20.0) \\
-/+\end{array}$ & $8(81.8)$ & & \\
- & $3(27.3)$ & $4(36.4)$ & 0.1984 & $4.7(0.77,28.5), 0.0950$ \\
\hline
\end{tabular}

E-cadherin, epithelial cadherin; AML, angiomyolipoma; CI, confidence intervals; EAML, epithelioid angiomyolipoma; OR, odds ratio; + , positive; -, negative.

differences in the proportion of epithelioid cell and antibody clone of E-cadherin may be another reason for the differences between the results of the two studies. Additionally, the present study investigated the association between E-cadherin and other biomarkers which have been identified in AML $(5,38)$. The results of the present study demonstrated that the expression of E-cadherin may be positively associated with the expression of HMB45 and Mart1/Melan A which are common biomarkers of melanomas $(\mathrm{P}<0.05)$. Tumors which expressed E-cadherin exhibited a decreased level of SMA and PR; however, no statistically significant difference was identified $(\mathrm{P}>0.05)$. In a previous study, Barnes et al (19) revealed that loss of E-cadherin expression may upregulate the expression of SMA in TSC2 gene (-/-) cell lines and is the only study, to the best of our knowledge, which has focused on the association between E-cadherin and other biomarkers. These cell lines were confirmed to exhibit a loss of the wild-type allele for the Tsc2 locus. In the present study, the association between E-cadherin and other biomarkers was observed at the tissue level; however, the underlying molecular mechanism and significance remains unknown. To elucidate this, a mechanistic study based on cell lines is required.

Secondly, the subcellular localization and staining category of E-cadherin in EAML and its control (triphasic AML) were investigated in the present study. The subcellular localization of E-cadherin was demonstrated to be significantly different between EAML and triphasic AML. Staining of the former demonstrated membranous (with or without cytoplasmic) E-cadherin, whereas triphasic AML revealed cytoplasmic E-cadherin staining only. In previous studies, E-cadherin exhibited a different staining intensity, a distinct localization between spindle and epithelioid cells in AML and staining identified cell membrane E-cadherin $(20,21)$, which is consistent with the results of the present study. In addition, the results of the study by Barnes et al (19) were consistent with those of the present study at the cell line level. The study by Barnes et al (19) demonstrated that wild-type rat renal epithelial cells exhibited plasma membrane staining of E-cadherin which aided cells to maintain the epithelioid characteristics. In contrast, the TSC2 (-/-) renal epithelial tumor-derived cells demonstrated a paucity of membrane E-cadherin and cells lost epithelioid morphology. In a previous study, Yang et al (39) hypothesized that increased expression of E-cadherin may be a critical step in MET. The results of the present study indicated that the various subcellular localizations of E-cadherin observed between EAML and triphasic AML may be one reason for the differences in cellular morphology between these two types of tumor.

Finally, the results of the present study identified differences between EAML cases possessing a low risk of exhibiting malignant behavior and EAML cases possessing intermediate and high risk of exhibiting malignant behavior. The cases categorized as low risk exhibited at least intermediate staining of E-cadherin, whereas the cases categorized as intermediate and high risk exhibited the opposite. Despite this, due to the limited number of cases, the significance of the association between expression of E-cadherin and malignant behavior remains unclear. Further studies are required to investigate this potential association.

The present case-control study was conducted to obtain more EAML cases retrospectively in a relatively short period. On the basis of current knowledge, it is understood that there are a number of risk factors for EAML; however, the only accepted risk factor was TSC (4,5). All the EAML cases had no history of TSC so only cases of triphasic AML without TSC were selected as a control. Additionally, the present study selected cases where patients also possessed common risk factors for cancer including a history of smoking and alcohol consumption. Although the cases with a strict condition were matched to guarantee the comparability between EAML and triphasic AML, there may be some confounding factors not identified in the present study which cannot be resolved using a case-control study. The small sample size and the heterogeneity in the proportion of ECs in EAML group were other limitations of the present study. However, EAML was a rare variant of AML and the present study established relative strict criteria of EAML and triphasic AML, on the basis of World Health Organization classification and the literature. The present study aimed for more suitable cases of EAML and triphasic AML to be recruited which resulted in all but 2 cases of EAML demonstrating $\geq 90 \%$ of ECs, which led to minimal heterogeneity. A study with a larger sample size from multiple centers and a relative homogeneity of the proportion 
of epithelioid cells in EAML, is required to increase the reliability of the results.

E-cadherin may an important biomarker in AML. The mechanisms of E-cadherin may be distinct between EAML and triphasic AML, since the subcellular localization of E-cadherin may be distinct between EAML and triphasic AML. A study with a large sample size is required to validate the results of the present study, followed by a mechanistic study based on cell lines to determine any significance.

\section{Acknowledgements}

The authors thank Professor Xiaohong R. Yang from the Genetic Epidemiology Branch, Division of Cancer Epidemiology and Genetics, National Cancer Institute, National Institutes of Health (Bethesda, MD, USA) for advice; Dr Zheng Yuan (Z.Y.) from the Department of Pathology, National Cancer Center/Cancer Hospital, Chinese Academy of Medical Sciences and Peking Union Medical College for assistance with reviewing the hematoxylin and eosin slides of the 22 cases; Dr Wei-Hua Li from the Department of Pathology, National Cancer Center/Cancer Hospital, Chinese Academy of Medical Sciences and Peking Union Medical College for assistance with preparing figures; and Mr Jiang-Nan Shao from the Fourth Educational Department of Beijing Li Li Changping Education Technology Co., Ltd. (Beijing, China) for assistance in native language revision. The present study was supported by the Beijing Hope Run Special Fund (grant no. LC2011B35) and the Basic Scientific Research Fund of China Central Public Welfare (grant no. JK 2014B06).

\section{References}

1. Hassan M,El-Hefnawy AS, Elshal AM, Mosbah A, El-Baz M and Shaaban A: Renal epithelioid angiomyolipoma: A rare variant with unusual behavior. Int Urol Nephrol 46: 317-322, 2014.

2. Koo KC, Kim WT, Ham WS, Lee JS, Ju HJ and Choi YD: Trends of presentation and clinical outcome of treated renal angiomyolipoma. Yonsei Med J 51: 728-734, 2010.

3. Lane BR, Aydin H, Danforth TL, Zhou M, Remer EM, Novick AC and Campbell SC: Clinical correlates of renal angiomyolipoma subtypes in 209 patients: Classic, fat poor, tuberous sclerosis associated and epithelioid. J Urol 180: 836-843. 2008.

4. Rakowski SK, Winterkorn EB, Paul E, Steele DJ, Halpern EF and Thiele EA: Renal manifestations of tuberous sclerosis complex: Incidence, prognosis, and predictive factors. Kidney Int 70: 1777-1782, 2006.

5. Eble JN, Sauter G, Epstein J and Sesterhenn IA (Eds): World Health Organization Classification of Tumours. Pathology and Genetics of Tumours of the Urinary System and Male Genital Organs. IARC Press, Lyon, pp65-69, 2004.

6. Mai KT, Perkins DG and Collins JP: Epithelioid cell variant of renal angiomyolipoma. Histopathology 28: 277-280, 1996.

7. Brimo F, Robinson B, Guo C, Zhou M, Latour M and Epstein JI: Renal epithelioid angiomyolipoma with atypia: A series of 40 cases with emphasis on clinicopathologic prognostic indicators of malignancy. Am J Surg Pathol 34: 715-722, 2010.

8. Nese N, Martignoni G, Fletcher CD, Gupta R, Pan CC, Kim H, Ro JY, Hwang IS, Sato K, Bonetti F, et al: Pure epithelioid PEComas (so-called epithelioid angiomyolipoma) of the kidney: A clinicopathologic study of 41 cases: Detailed assessment of morphology and risk stratification. Am J Surg Pathol 35: 161-176, 2011.

9. Srigley JR, Delahunt B, Eble JN, Egevad L, Epstein JI, Grignon D, Hes O, Moch H, Montironi R, Tickoo SK, et al: The international society of urological pathology (ISUP) vancouver classification of renal neoplasia. Am J Surg Pathol 37: 1469-1489, 2013.
10. Martignoni G, Pea M, Rigaud G, Manfrin E, Colato C, Zamboni G, Scarpa A, Tardanico R, Roncalli M and Bonetti F: Renal angiomyolipoma with epithelioid sarcomatous transformation and metastases: Demonstration of the same genetic defects in the primary and metastatic lesions. Am J Surg Pathol 24: $889-894,2000$

11. Sato K, Ueda Y, Tachibana H, Miyazawa K, Chikazawa I, Kaji S, Nojima T and Katsuda S: Malignant epithelioid angiomyolipoma of the kidney in a patient with tuberous sclerosis: An autopsy case report with p53 gene mutation analysis. Pathol Res Pract 204: 771-777, 2008

12. Bing Z, Yao Y, Pasha T, Tomaszewski JE and Zhang PJ: p53 in pure epithelioid PEComa: An immunohistochemistry study and gene mutation analysis. Int J Surg Pathol 20: 115-122, 2012 .

13. Li J, Zhu M and Wang YL: Malignant epithelioid angiomyoliopoma of the kidney with pulmonary metastases and p53 gene mutation. World J Surg Oncol 10: 213, 2012.

14. Li W, Guo L, Bi X, Ma J and Zheng S: Immunohistochemistry of p53 and Ki-67 and p53 mutation analysis in renal epithelioid angiomyolipoma. Int J Clin Exp Pathol 8: 9446-9451, 2015.

15. Ooi SM, Vivian JB and Cohen RJ: The use of the Ki-67 marker in the pathological diagnosis of the epithelioid variant of renal angiomyolipoma. Int Urol Nephrol 41: 559-565, 2009.

16. Thiery JP: Epithelial-mesenchymal transitions in development and pathologies. Curr Opin Cell Biol 15: 740-746, 2003.

17. Yang $\mathrm{J}$ and Weinberg RA: Epithelial-mesenchymal transition: At the crossroads of development and tumor metastasis. Dev Cell 14: 818-829, 2008.

18. Thiery JP, Acloque H, Huang RY and Nieto MA: Epithelial-mesenchymal transitions in development and disease. Cell 139: 871-890, 2009.

19. Barnes EA, Kenerson HL, Jiang X and Yeung RS: Tuberin regulates E-cadherin localization: Implications in epithelial-mesenchymal transition. Am J Pathol 177: 1765-1778, 2010.

20. Wang Z, Gong Q and Fan Q: Expression of E-cadherin in angiomyolipoma. Hum Pathol 43: 2348-2353, 2012.

21. Konosu-Fukaya S, Nakamura Y, Fujishima F, Kasajima A, McNamara KM, Takahashi Y, Joh K, Saito H, Ioritani N, Ikeda Y, et al: Renal epithelioid angiomyolipoma with malignant features: Histological evaluation and novel immunohistochemical findings. Pathol Int 64: 133-141, 2014.

22. Alì G, Boldrini L, Capodanno A, Pelliccioni S, Servadio A, Crisman G, Picchi A, Davini F, Mussi A and Fontanini G: Expression of p-AKT and p-mTOR in a large series of bronchopulmonary neuroendocrine tumors. Exp Ther Med 2: 787-792, 2011.

23. Li N, Zhong M and Song M: Expression of phosphorylated mTOR and its regulatory protein is related to biological behaviors of ameloblastoma. Int J Clin Exp Pathol 5: 660-667, 2012.

24. Zheng S, Bi XG, Song QK, Yuan Z, Guo L, Zhang H and Ma JH: A suggestion for pathological grossing and reporting based on prognostic indicators of malignancies from a pooled analysis of renal epithelioid angiomyolipoma. Int Urol Nephrol 47: 1643-1651,2015.

25. Lecuit T and Yap AS: E-cadherin junctions as active mechanical integrators in tissue dynamics. Nat Cell Biol 17: 533-539, 2015.

26. Takeichi M: Dynamic contacts: Rearranging adherens junctions to drive epithelial remodelling. Nat Rev Mol Cell Biol 15: 397-410, 2014.

27. Gallin WJ, Edelman GM and Cunningham BA: Characterization of L-CAM, a major cell adhesion molecule from embryonic liver cells. Proc Natl Acad Sci USA 80: 1038-1042, 1983.

28. Schuh R, Vestweber D, Riede I, Ringwald M, Rosenberg UB Jäckle $\mathrm{H}$ and Kemler R: Molecular cloning of the mouse cell adhesion molecule uvomorulin: cDNA contains a B1-related sequence. Proc Natl Acad Sci USA 83: 1364-1368, 1986.

29. Ogou SI, Yoshida-Noro C and Takeichi M: Calcium-dependent cell-cell adhesion molecules common to hepatocytes and teratocarcinoma stem cells. J Cell Biol 97: 944-948, 1983.

30. Yoshida-Noro C, Suzuki N and Takeichi M: Molecular nature of the calcium-dependent cell-cell adhesion system in mouse teratocarcinoma and embryonic cells studied with a monoclonal antibody. Dev Biol 101: 19-27, 1984.

31. Mertz AF, Che Y, Banerjee S, Goldstein JM, Rosowski KA, Revilla SF, Niessen CM, Marchetti MC, Dufresne ER and Horsley V: Cadherin-based intercellular adhesions organize epithelial cell-matrix traction forces. Proc Natl Acad Sci USA 110: 842-847, 2013. 
32. Ghahhari NM and Babashah S: Interplay between microRNAs and $\mathrm{WNT} / \beta$-catenin signalling pathway regulates epithelial-mesenchymal transition in cancer. Eur $\mathrm{J}$ Cancer 51: $1638-1649,2015$.

33. Bhatt T, Rizvi A, Batta SP, Kataria S and Jamora C: Signaling and mechanical roles of E-cadherin. Cell Commun Adhes 20: 189-199, 2013.

34. Kobayashi N, Matsuzaki O, Shirai S, Aoki I, Yao M and Nagashima Y: Collecting duct carcinoma of the kidney: An immunohistochemical evaluation of the use of antibodies for differential diagnosis. Hum Pathol 39: 1350-1359, 2008.

35. Muramaki M, Miyake H, Terakawa T, Kusuda Y and Fujisawa M: Expression profile of E-cadherin and $\mathrm{N}$-cadherin in urothelial carcinoma of the upper urinary tract is associated with disease recurrence in patients undergoing nephroureterectomy. Urology 78: 1443.e7-e12, 2011.
36. Wang N, He YL, Pang LJ, Zou H, Liu CX, Zhao J, Hu JM, Zhang WJ, Qi Y and Li F: Down-regulated E-cadherin expression is associated with poor five-year overall survival in bone and soft tissue sarcoma: Results of a meta-analysis. PLoS One 10: e0121448, 2015.

37. Yoo J, Park S, Kang CS, Kang SJ and Kim BK: Expression of E-cadherin and p53 proteins in human soft tissue sarcomas. Arch Pathol Lab Med 126: 33-38, 2002.

38. Martignoni G, Pea M, Reghellin D, Zamboni G and Bonetti F: PEComas: The past, the present and the future. Virchows Arch 452: 119-132, 2008

39. Yang J, Du X, Wang G, Sun Y, Chen K, Zhu X, Lazar AJ, Hunt KK, Pollock RE and Zhang W: Mesenchymal to epithelial transition in sarcomas. Eur J Cancer 50: 593-601, 2014. 